\title{
Using CSW weight's in UTASTAR method
}

\author{
Ahmad Makui* and Maryam Momeni
}

Department of Industrial Engineering, Iran University of Science and Technology, Tehran, Iran

\begin{tabular}{|c|c|}
\hline A R T I L E I N F O & A B S T R A T T \\
\hline $\begin{array}{l}\text { Article history: } \\
\text { Received March 29, } 2012 \\
\text { Accepted } 18 \text { June } 2012 \\
\text { Available online } \\
\text { June } 192012 \\
\text { Keywords: } \\
\text { CSW } \\
\text { UTASTAR } \\
\text { DEA }\end{array}$ & $\begin{array}{l}\text { Several researchers have considered similarities between Multi-Criteria Decision Making } \\
\text { (MCDM) and Data Envelopment Analysis (DEA), as tools for solving decision making } \\
\text { problems. As the preferences of decision- maker (DM) on alternatives are not considered in } \\
\text { classical DEA, some researchers have tried to consider it in DEA. } \\
\text { The UTA-STAR method is one of the techniques widely used in Multi Criteria Decision } \\
\text { Analysis. In this technique, the preferences of decision maker on alternatives are considered } \\
\text { and UTA-STAR tries to compute the most suitable weights for criteria and alternatives to } \\
\text { obtain a utility function having a minimum deviation from the preferences. The goal of this } \\
\text { paper is interpreting decision maker's preferences in UTA-STAR method, in a new manner, } \\
\text { using the common set of weights (CSW) in DEA. }\end{array}$ \\
\hline
\end{tabular}

(c) 2012 Growing Science Ltd. All rights reserved.

\section{Introduction}

Late of 1970's was the starting point of creating DEA and it has been used to measure the efficiency of decision making units with multiple inputs and multiple outputs. DEA uses linear programming as its computational tool (Jahanshahloo et al., 2005).

One of the important philosophical differentiates between DEA and MCDM is that MCDM is generally faced with human judgment. Unlike MCDM, DEA seeks to extract identical data and avoiding from this judgment. These two fields shall learn various issues from each other: Decision making models, having standard multi criteria, are performed, carefully, and do not consider decision maker judgment. On the other hand, DEA may use performed tasks in MCDM, for extracting these judgment, especially, putting bound for weights (Azadeh et al., 2008).

During recent decade, successful implementation of DEA in part of performance measurement has been a reason for the usage of DEA as a tool for MCDM. In this concept, decision making units are replaced with alternatives, inputs are replaced with profit criteria, which must be maximized and the outputs are replaced by cost criteria, which must be minimized (Makui et al., 2008). Golany

\footnotetext{
* Corresponding author. Tel: +9892177240000

E-mail addresses: amakui@iust.ac.ir (A. Makui) 
performed the first attempts for combining DEA and multi objective linear programming and Stewart compared the classic goals of DEA and MCDM (Mavrotas \& Trifillis, 2006). The most important aim of "DEA" is to recognize efficiency score of a system or decision making unit, by comparison between these units, whereas, the goal of MCDM is to rank and choose a set of alternatives using multiple criteria (Ramanathan, 2006). Many of the researchers have combined DEA and MCDM techniques. For example, Azadeh et al. (2008) used AHP for estimating the weights of criteria. Tseng and Lee (2005) proposed DEA/AHP method for measuring organizational performance. Another authors indicated, it is possible to use ranking model of "AHP/DEA", for the purpose of improving the capacity of using DEA (Ramanathan, 2006). Wang et al. (2008) suggested a DEA model with assurance region (AR), for priority derivation in AHP, named "DEA/ AR". Amiri et al. (2010) introduced an integrated DEA-TOPSIS methodology for portfolio risk evaluation. The other people used multiple criteria approaches to DEA (Li, \& Reeves, 1999). Hosseinzadeh et al. (2009) investigated the relationship between DEA and Multi Objective Decision Making. Makui \& et al. (2008) proposed using a multiple objective linear programming approach for determining common set of weights (CSW) in DEA. Soares De mello et al. (2002) combined two methods, both DEA and MACBETH.

Lack of preferences of decision maker was one of the weakness of DEA in past. It has been considered as a significant advantage, in comparison with other decision-making methods and classical multi-criteria methods (Mavrotas \& Trifillis, 2006). Actually, DEA is associated with MCDM tools, for making decisions about multi dimensional alternatives. According to Sarkis (2000), it seems that DEA is considered as a MCDM tool, having discrete alternative multiple criteria decision information. In DEA, decision maker's judgments are interfered in two ways. At first, these judgments have been studied in relation with criteria through elicitation of utilities for various levels of criteria values. Generally, it is focused on criteria data, not DEA formulations. The simple way is to add constraints and re-model the structure of preferences of decision maker (Sarkis, 2000).

\section{Common weights \& DEA}

The idea of common weights in "DEA" was first introduced for considering DEA for measuring maintenance units of highways and it was presented as mental preferences in DEA framework (Sinuany-Stern \& Friedman, 1998).

Common weights have been used for ranking data. For example, some people considered common set of weights for ranking decision making units and maximized the sum of their efficiency (Wang et al., 2006). The other people introduced a two-stage linear discriminate analysis approach to generate common set of weights. They performed canonic correlation analysis for receiving a weight vector, for all inputs and outputs. On the other hand, they introduced non-linear discriminate analysis, measuring common weights for all related units (Sinuany-Stern \& Friedman, 1998). In addition, some other people used common weights for measuring the exact efficiency of ranked units based on DEA (Wang et al., 2006). In several researches, some frameworks have been offered about putting desired bounds in DEA and considered set of common weights (Jahanshahloo et al., 2005). In Liu and Peng's study, a method is suggested, determining the set of common weights for standards of efficient decision- making unit's performance in case of DEA. Then, these units were ranked according to score of efficiency of considered weights (Liu \& Peng, 2008). In their study, a process is performed, for the purpose of obtaining robust ranking, by correcting region bounds and other Limitation of artificial weights in any measurement (Liu \& Peng, 2009).

CSW is formulated for determining common weights of $\underline{\underline{n}}$ units, having $\underline{\underline{m}}$ inputs and $\underline{\underline{s}}$ outputs, as follows, 


$$
\begin{array}{ll}
\Delta^{*}= & \min \sum_{j=1}^{n}\left(\Delta_{j}^{O}+\Delta_{j}^{I}\right) \\
\text { s.t. } & \frac{\sum_{\mathrm{r}=1}^{\mathrm{s}} \mathrm{y}_{\mathrm{rj}} U_{r}+\Delta_{j}^{O}}{\sum_{i=1}^{m} x_{i j} V_{i}-\Delta_{j}^{I}}=1, \quad j=1, \ldots, n \\
& \Delta_{j}^{O}, \Delta_{j}^{I} \geq 0, j=1, \ldots, n \\
& U_{r} \geq \varepsilon \succ 0, r=1, \ldots, s \\
& V_{j \geq} \varepsilon \succ 0, i=1, \ldots, m .
\end{array}
$$

$x_{i j}$ is the value of input index $i$ of the $j^{\text {th }}$ DMU, $\mathrm{y}_{\mathrm{rj}}$ is the value of output index $r$ of $j^{\text {th }} \mathrm{DMU}, \mathrm{V}_{\mathrm{i}}$ the common weights for all DMUs attached to the input index $i$, and the $\mathrm{U}_{\mathrm{r}}$ is the common weights for all DMUs attached to output index $r$.

Optimized common weights set of $\mathrm{u}_{\mathrm{r}}(\mathrm{r}=1,2, \ldots, \mathrm{s})$ and $\mathrm{v}_{\mathrm{i}}(\mathrm{i}=1,2$, , ,, , $\mathrm{m})$ are used for recognizing the exact score of unit and ranking them.

\section{UTA-STAR technique}

In MCDM literature, several methods have been delivered for determining the weights of alternatives. Generally, they are divided in to three parts, including: subjective, objective and integrated. Subjective methods determine the weights of alternatives, according to subjective preference of decision maker, including LINMAP (Linear Programming Techniques for Multidimensional Analysis of Preferences) (Wang \& Luo, 2010).

UTA (Utilites Additives) method has been stated by Jacquet-Lagreze and Siskos (1982). It extracts one or more additive value functions from a given ranking on a reference alternatives $A_{R}$ set. Its input is faced with a set of $A_{R}$ options. On the other hand, it is asked from decision maker to introduce his general value making among $A_{R}$ alternatives. For obtaining utility functions, UTA uses linear programming technique. Finally, "UTASTAR” has been considered by Siskos \& Yannacopoulos in 1985, being as a corrected version of main UTA model.

The mathematical model used in UTA-STAR is as following:

$[\min ] z=\sum_{k=1}^{m}\left[\sigma^{+}\left(a_{k}\right)+\sigma^{-}\left(a_{k}\right)\right]$

subject to

$$
\begin{aligned}
& \left.\begin{array}{l}
\Delta\left(a_{k}, a_{k+1}\right) \geq \delta \text { if } a_{k} \succ a_{k+1} \\
\Delta\left(a_{k}, a_{k+1}\right)=\delta \text { if } a_{k} \approx a_{k+1}
\end{array}\right\} \forall k \\
& \sum_{i=1}^{n} \sum_{j=1}^{a_{i}-1} w_{i j} \\
& w_{i j} \geq 0, \sigma^{+}\left(a_{k}\right) \geq 0, \sigma^{-}\left(a_{k}\right) \geq 0 \quad \forall i, j, k \\
& u^{\prime}[g(a)]=\sum_{i=1}^{n} u_{i}\left[g\left(a_{k}\right)\right]-\sigma^{+}\left(a_{k}\right)+\sigma^{-}\left(a_{k}\right) \quad \forall a \in A_{R} \\
& \Delta\left(a_{k}, a_{k+1}\right)=u\left[g\left(a_{k}\right)\right]-\sigma^{+}\left(a_{k}\right)+\sigma^{-}\left(a_{k}\right)-u\left[g\left(a_{k+1}\right)\right]+\sigma^{+}\left(a_{k+1}\right)-\sigma^{-}\left(a_{k+1}\right) .
\end{aligned}
$$


In Model (2), $a_{k}$ and $a_{k+1}$ are alternatives in the set $A_{R} \cdot w_{i j} s$ are the weights assigned to interval values of criteria. The constraints present the preferences of decision maker and the objective function minimizes the deviations of the utility function $U$ proposed by the model and the utility function hidden in the preferences of the alternatives in the set $A_{R}$.

\section{Suggested model}

According to performed literature review in field of making a relation between DEA and MCDM, there has been no link between DEA and UTA-STAR technique, for facilitating using CSW, as suggested in present paper. Actually, decision maker preferences on considered alternatives of CSW model is done by a new method and adding a new criterion. This kind of criterion is cost or an output. The preferences of decision maker are shown by a new constraint in the CSW model. For using these weights in UTASAR, the weight of new criterion must be omitted and divided relatively among other weights. Finally, the weights of main criteria must be normalized. For considering these weights in UTASTAR, several constraints, must be added in to the model.

The steps of the method can be summarized as follows:

1) Consider the profit criteria as the inputs and the cost criteria as the outputs of DEA method,

2) By using the CSW model (1), find the common set of weights,

3) Consider the preferences on the alternatives, suggested by decision maker, as a new output in DEA model (consider the ranking of alternatives),

4) Omit the weights of the new output, considered in step 3, and divide its value relatively between the other inputs and outputs,

5) Use the obtained weights in UTASTAR model (2).

\section{Numerical example}

Following example is related to the selecting a supplier from 10 candidates. The data are extracted from paper (Tseng \& Lee, 2009). Cost and lead time criteria are considered as cost criteria and are inputs. Quality, Quantity, technology, services and direct delivery in to necessary places are considered as profit and are outputs. It is assumed that preferences of decision maker are ranked on these 10 alternatives, in following table:

\section{Table 1}

Data for numerical example

\begin{tabular}{ccccccccc}
\hline Rank & Alternative & Price & Quality & Lead time & Quantity & Delivery & Technology & Service \\
\hline 1 & A & 8.75 & 7 & 3 & 6 & 6 & 3 & 5 \\
10 & B & 7.25 & 2 & 2 & 4 & 4 & 4 & 4 \\
7 & C & 10.24 & 6 & 4 & 2 & 7 & 3 & 4 \\
6 & D & 11.28 & 6 & 2 & 1 & 6 & 7 & 5 \\
4 & E & 11.2 & 7 & 2 & 4 & 3 & 7 & 6 \\
3 & F & 11.73 & 7 & 1 & 4 & 6 & 4 & 7 \\
9 & G & 6 & 3 & 4 & 5 & 4 & 5 & 4 \\
5 & H & 9.77 & 5 & 2 & 7 & 1 & 5 & 6 \\
8 & I & 11.1 & 6 & 3 & 3 & 2 & 7 & 4 \\
2 & J & 8.91 & 5 & 3 & 7 & 7 & 4 & 6 \\
\hline
\end{tabular}

For interfering to these kinds of preferences in CSW model, $\mathrm{U}_{6}$ is considered as a new output variable. 
$\min D_{1}+D_{2}+D_{3}+D_{4}+D_{5}+D_{6}+D_{7}+D_{8}+D_{9}+D_{10}$

subject to

$$
\begin{aligned}
& 7 U_{1}+6 U_{2}+6 U_{3}+3 U_{4}+5 U_{5}-8.75 v_{1}-3 V_{2}+9 U_{6}+D_{1}=0 \\
& 2 U_{1}+4 U_{2}+4 U_{3}+4 U_{4}+4 U_{5}-7.25 V_{1}-2 V_{2}+D_{2}=0 \\
& 6 U_{1}+2 U_{2}+7 U_{3}+3 U_{4}+4 U_{5}-10.24 V_{1}-4 V_{2}+3 U_{6}+D_{3}=0 \\
& 6 U_{1}+U_{2}+6 U_{3}+7 U_{4}+5 U_{5}-11.28 V_{1}-2 V_{2}+4 U_{6}+D_{4}=0 \\
& 7 U_{1}+4 U_{2}+3 U_{3}+7 U_{4}+6 U_{5}-11.2 V_{1}-2 V_{2}+6 U_{6}+D_{5}=0 \\
& 7 U_{1}+4 U_{2}+6 U_{3}+4 U_{4}+7 U_{5}-11.73 V_{1}-V_{2}+7 U_{6}+D_{6}=0 \\
& 3 U_{1}+5 U_{2}+4 U_{3}+5 U_{4}+4 U_{5}-6 V_{1}-4 V_{2}+U_{6}+D_{7}=0 \\
& 5 U_{1}+7 U_{2}+U_{3}+5 U_{4}+6 U_{5}-9.77 V_{1}-2 V_{2}+5 U_{6}+D_{8}=0 \\
& 6 U_{1}+3 U_{2}+2 U_{3}+7 U_{4}+4 U_{5}-11.1 V_{1}-3 V_{2}+2 U_{6}+D_{9}=0 \\
& 5 U_{1}+7 U_{2}+7 U_{3}+4 U_{4}+6 U_{5}-8.91 V_{1}-3 V_{2}+8 U_{6}+D_{10}=0 \\
& U_{k}>=0.01, k=1, \ldots, 6 \\
& V_{1}>=0.01 \\
& V_{2}>=0.01 \\
& D_{m}>=0, m=1, \ldots, 10
\end{aligned}
$$

In this Level, following weights have been obtained:

\begin{tabular}{cccccccc}
\hline$U_{1}$ & $U_{2}$ & $U_{3}$ & $U_{4}$ & $U_{5}$ & $U_{6}$ & $V_{1}$ & $V_{2}$ \\
\hline 0.01 & 0.01 & 0.01 & 0.029315 & 0.01 & 0.01 & 0.031755 & 0.054774 \\
\hline
\end{tabular}

The weight of new added criterion is omitted and divided among the remaining ones. Finally, these weights are normalized for using in UTASTAR model and we have following weights in this model:

\begin{tabular}{ccccccc}
\hline$U_{1}$ & $U_{2}$ & $U_{3}$ & $U_{4}$ & $U_{5}$ & $V_{1}$ & $V_{2}$ \\
\hline 0.066 & 0.066 & 0.066 & 0.186 & 0.066 & 0.203 & 0.347 \\
\hline
\end{tabular}

The efficiency of each alternative is obtained as price(11.73,10.24,9.77,7.25,6), quality(2,3,5,6,7), leadtime(4,3,2,1), quantity(1,3,5,7), delivery(1,3,5,7), technology(3,4,5,6,7) and services(4,5,6,7). More details are given in the appendix. Table 2 shows the results of both new and classic model of UTASTAR, including its utilities and ranking:

Table 2

The results of classical and UTASTAR methods

\begin{tabular}{ccccc}
\hline Alternative & Utility in classic model & Utility in new model & Ranking of classic model & Ranking of new model \\
\hline A & 0.7125 & 0.699 & 1 & 1 \\
B & 0.05 & 0.368 & 10 & 9 \\
C & 0.225 & 0.350 & 7 & 10 \\
D & 0.275 & 0.449 & 6 & 7 \\
E & 0.475 & 0.549 & 4 & 4 \\
F & 0.525 & 0.599 & 3 & 3 \\
G & 0.1 & 0.418 & 9 & 8 \\
H & 0.425 & 0.499 & 5 & 5 \\
I & 0.15 & 0.475 & 8 & 6 \\
J & 0.575 & 0.649 & 2 & 2 \\
\hline
\end{tabular}




\section{Conclusion}

In this paper, we have presented a method for making a relation between DEA and UTASTAR. By performing mentioned changes in classic CSW model, the new one has been considered by focusing on efficiency of decision making units of preferences. As can be seen in the numerical example, the difference between the classical method's ranking and the new method's ranking is not too high and so the proposed method can be considered as a suitable way for combining DEA and MCDM.

\section{Acknowledgment}

The authors would like to thank the anonymous referees for their constructive comments on earlier version of this work.

\section{References}

Amiri, M., Zandieh, M., Vahdani, B., Soltani, R., \& Roshanaei, V. (2010). An integrated eigenvector-DEA-TOPSIS methodology for portfolio risk evaluation in the FOREX spot market. Expert Systems with Applications, 37, 509-516.

Azadeh , A., Ghaderi , S.F., \& Izadbakhsh, H. (2008). Integration of DEA and AHP with computer simulation for railway system improvement and optimization. Applied Mathematics and Computation, 195, 775-785.

Hosseinzadeh Lotfi, F., \& Jahanshahloo, G.R., Soltanifar, M., Ebrahimnejad, A., Mansourzadeh, S.M. (2009). Relationship between MOLP and DEA based on output-orientated CCR dual model. Expert Systems with Applications , 37(6), 4331-4336.

Jahanshahloo, G.R., Memariani , A., Hosseinzadeh Lotfi, F., \& Rezai, H.Z. (2005). A note on some of DEA models and finding efficiency and complete ranking using common set of weights. Applied Mathematics and Computation, 166, 265-281.

Li, X.B., Reeves, G.R. (1999). A multiple criteria approach to data envelopment analysis. European Journal of Operational Research, 115, 507-517.

Liu, F.F.H., \& Peng, H.H. (2008). Ranking of units on the DEA frontier with common weights. Computers \& Operations Research, 35, 1624-1637,(2008)

Liu, F.F.H., \& Peng, H.H. (2009). A systematic procedure to obtain a preferable and robust ranking of units. Computers \& Operations Research, 36, 1012-1025.

Makui, A., Alinezhad , A., Kiani Mavi, R., \& Zohrehbandian, M. (2008). A goal programming method for finding common weights in DEA with an improved discriminating power for efficiency. Journal of Industrial and Systems Engineering, 1(4), 293-303.

Mavrotas, G. \& Trifillis, P. (2006). Multicriteria decision analysis with minimum information: combining DEA with MAVT. Computers \& Industrial Engineering, 33, 2083-2098.

Ramanathan, R. (2006). Data envelopment analysis for weight derivation and aggregation in the analytic hierarchy process. Computers \& Operations Research, 33, 1289-130

Sarkis, J. (2000). A comparative analysis of DEA as a discrete alternative multiple criteria decision tool. European Journal of Operational Research, 123(3), 543-557.

Soares de Mello, J.C.C.B., Lins, M.P.E., Soares de Mello, M.H.C., \& Gomes, E.G. (2002). Evaluating the performance of calculus classes using operational research tools. European Journal of Engineering Education, 27(2), 209-218.

Sinuany-Stern, Z., \& Friedman, L. (1998). DEA and the discriminant analysis of ratio for ranking units. European Journal of Operational Research, 111,470-478.

Tseng , Y.F., \& Lee, T.Z. (2009). Comparing appropriate decision support of human resource practices on organizational performance with DEA/AHP model. Expert Systems with Applications, 36, 6548-6558.

Wang, Y.M., Luo, Y., \& Liang, L. (2009). Ranking decision making units by imposing a minimum weight restriction in the data envelopment analysis. Journal of Computational and Applied Mathematics, 223 ,469-484. 
Wang, Y.M., Chin, K.S., \& Poon, G.K.K. (2008). A data envelopment analysis method with assurance region for weight generation in the analytic hierarchy process. Decision Support Systems, 45 , 913-921.

Wang, Y.M., \& Luo, Y. (2010). Integration of correlations with standard deviations for determining attribute weights in multiple attribute decision making, Mathematical and Computer Modelling, 51(1-2), 1-12.

\section{Appendix}

The new UTASTAR model is as the following:

$\min$

$$
\begin{aligned}
& D_{1 A}+D_{2 A}+D_{1 B}+D_{2 B}+D_{1 C}+D_{2 C}+D_{1 D}+D_{2 D}+D_{1 E}+D_{2 E}+D_{1 F}+D_{2 F}+D_{1 G}+D_{2 G}+D_{1 H}+ \\
& D_{2 H}+D_{1 I}+D_{2 I}+D_{1 J}+D_{2 J}
\end{aligned}
$$

subject to

$$
\begin{aligned}
& W_{11}+W_{12}+0.404 W_{13}+W_{21}+W_{22}+W_{23}+W_{24}+W_{31}+W_{41}+W_{42}+0.5 W_{43}+W_{51}+W_{52}+0.5 W_{53} \\
& +W_{71}-W_{11}-W_{12}-0.341 W_{13}-W_{21}-W_{22}-W_{31}-W_{41}-W_{42}-W_{43}-W_{51}-W_{52}-W_{53}-W_{61}-W_{71}- \\
& W_{72}-D_{1 A}+D_{2 A}+D_{1 J}-D_{2 J} \geq 0.05 \\
& W_{11}+W_{12}+0.341 W_{13}+W_{21}+W_{22}+W_{31}+W_{41}+W_{42}+W_{43}+W_{51}+W_{52}+W_{53}+W_{61}+W_{71}+W_{72}- \\
& W_{21}-W_{22}-W_{23}-W_{24}-W_{31}-W_{32}-W_{33}-W_{41}-0.5 W_{42}-W_{51}-W_{52}-0.5 W_{53}-W_{61}-W_{71}-W_{72}- \\
& W_{73}-D_{1 J}+D_{2 J}+D_{1 F}-D_{2 F} \geq 0.05 \\
& W_{21}+W_{22}+W_{23}+W_{24}+W_{31}+W_{32}+W_{33}+W_{41}+0.5 W_{42}+W_{51}+W_{52}+0.5 W_{53}+W_{61}+W_{71}+W_{72}+ \\
& W_{73}-0.355 W_{11}-W_{21}-W_{22}-W_{23}-W_{24}-W_{31}-W_{32}-W_{41}-0.5 W_{42}-W_{51}-W_{61}-W_{62}-W_{63}-W_{64}- \\
& W_{71}-W_{72}-D_{1 F}+D_{2 F}+D_{1 E}-D_{2 E} \geq 0.05 \\
& 0.355 W_{11}+W_{21}+W_{22}+W_{23}+W_{24}+W_{31}+W_{32}+W_{41}+0.5 W_{42}+W_{51}+W_{61}+W_{62}+W_{63}+W_{64}+ \\
& W_{71}+W_{72}-W_{11}-W_{12}-W_{21}-W_{22}-W_{31}-W_{32}-W_{41}-W_{42}-W_{43}-W_{61}-W_{62}-W_{71}-W_{72}- \\
& D_{1 E}+D_{2 E}+D_{1 H}-D_{2 H} \geq 0.05 \\
& W_{11}+W_{12}+W_{21}+W_{22}+W_{31}+W_{32}+W_{41}+W_{42}+W_{43}+W_{61}+W_{62}+W_{71}+W_{72}-0.302 W_{11}- \\
& W_{21}-W_{22}-W_{23}-W_{31}-W_{32}-W_{51}-W_{52}-0.5 W_{53}-W_{61}-W_{62}-W_{63}-W_{64}-W_{71}-D_{1 H}+D_{2 H}+D_{1 D}- \\
& D_{2 D} \geq 0.05 \\
& 0.302 W_{11}+W_{21}+W_{22}+W_{23}+W_{31}+W_{32}+W_{51}+W_{52}+0.5 W_{53}+W_{61}+W_{62} \\
& +W_{63}+W_{64}+W_{71}-W_{11}-W_{21}-W_{22}-W_{23}-0.5 W_{41}-W_{51}-W_{52}-W_{53}-D_{1 D}+D_{2 D}+D_{1 C}-D_{2 C} \geq 0.05 \\
& W_{11}+W_{21}+W_{22}+W_{23}+0.5 W_{41}+W_{51}+W_{52}+W_{53}-0.423 W_{11}-W_{21}-W_{22}-W_{23}- \\
& W_{31}+W_{41}+0.5 W_{51}-W_{61}-W_{62}-W_{63}-W_{64}-D_{1 C}+D_{2 C}+D_{1 I}-D_{2 I} \geq 0.05
\end{aligned}
$$


$+W_{63}+W_{64}+W_{71}-W_{11}-W_{21}-W_{22}-W_{23}-0.5 W_{41}-W_{51}-W_{52}-W_{53}-D_{1 D}+D_{2 D}+D_{1 C}-D_{2 C} \geq 0.05$

$W_{11}+W_{21}+W_{22}+W_{23}+0.5 W_{41}+W_{51}+W_{52}+W_{53}-0.423 W_{11}-W_{21}-W_{22}-W_{23}-$

$W_{31}+W_{41}+0.5 W_{51}-W_{61}-W_{62}-W_{63}-W_{64}-D_{1 C}+D_{2 C}+D_{1 I}-D_{2 I} \geq 0.05$

$0.423 w_{11}+w_{21}+w_{22}+w_{23}+w_{31}+w_{41}+0.5 w_{51}+w_{61}+w_{62}+w_{63}+w_{64}-w_{11}-w_{12}-w_{13}-$

$w_{21}-w_{41}-w_{42}-w_{51}-0.5 w_{52}-w_{61}-w_{62}-D_{1 I}+D_{2 I}+D_{1 G}-D_{2 G} \geq 0.05$

$w_{11}+w_{12}+w_{13}+w_{21}+w_{41}+w_{42}+w_{51}+0.5 w_{52}+w_{61}+w_{62}-w_{11}-w_{12}-w_{13}-w_{31}-w_{32}-$

$w_{41}-0.5 w_{42}-w_{51}-0.5 w_{52}-w_{61}-D_{1 G}+D_{2 G}+D_{1 B}-D_{2 B} \geq 0.05$

$w_{11}+w_{12}+w_{13}+w_{14}=0.203$

$w_{21}+w_{22}+w_{23}+w_{24}=0.066$

$w_{31}+w_{32}+w_{33}=0.347$

$w_{41}+w_{42}+w_{43}=0.066$

$w_{51}+w_{52}+w_{53}=0.066$

$w_{61}+w_{62}+w_{63}+w_{64}=0.186$

$w_{71}+w_{72}+w_{73}=0.066$

$w_{11}, w_{12}, w_{13}, w_{14}, w_{21}, w_{22}, w_{23}, w_{24}, w_{31}, w_{32}, w_{33}, w_{41}, w_{42}, w_{43}, w_{51}, w_{52}, w_{53}$,

$w_{61}, w_{62}, w_{63}, w_{64}, w_{71}, w_{72}, w_{73}, D_{1 A}, D_{2 A}, D_{1 B}, D_{2 B}, D_{1 C}, D_{2 C}, D_{1 D}, D_{2 D}, D_{1 E}, D_{2 E}$,

$D_{1 F}, D_{2 F}, D_{1 G}, D_{2 G}, D_{1 H}, D_{2 H}, D_{1 I}, D_{2 I}, D_{1 J}, D_{2 J} \geq 0$ 